\title{
Wavefront sensorless adaptive optics for optical coherence tomography guided femtosecond laser surgery in the posterior eye
}

Zabic, Miroslav, Matthias, Ben, Hahn, Jan, Heisterkamp, Alexander, Meyer, Heiko, et al.

Miroslav Zabic, Ben Matthias, Jan Hahn, Alexander Heisterkamp, Heiko Meyer, Tammo Ripken, "Wavefront sensorless adaptive optics for optical coherence tomography guided femtosecond laser surgery in the posterior eye," Proc. SPIE 10886, Adaptive Optics and Wavefront Control for Biological Systems V, 1088603 (20 February 2019); doi: 10.1117/12.2509363

SPIE. Event: SPIE BiOS, 2019, San Francisco, California, United States 


\title{
Wavefront sensorless adaptive optics for optical coherence tomography guided femtosecond laser surgery in the posterior eye
}

\author{
Miroslav Zabic ${ }^{\# * a, b}$, Ben Matthias ${ }^{\# a}$, Jan Hahn ${ }^{\mathrm{a}}$, Alexander Heisterkamp ${ }^{\mathrm{b}}$, Heiko Meyer ${ }^{\mathrm{a}}$, and \\ Tammo Ripken ${ }^{\mathrm{a}}$ \\ ${ }^{a}$ Industrial and Biomedical Optics Department, Laser Zentrum Hannover e.V., Hollerithallee 8, \\ 30419 Hannover, Germany \\ ${ }^{\mathrm{b}}$ Institute of Quantum Optics, Leibniz University Hannover, Welfengarten 1, 30167 Hannover, \\ Germany
}

\begin{abstract}
Surgery with fs-laser in the posterior part of the eye could be useful for separation of tractional epiretinal membrane and vitreous floaters treatment. However, focus degradation occurs near the retina due to induced aberrations by cornea and lens. To overcome this issue, adaptive optics with wavefront sensor and wavefront modulator can be utilized. We demonstrate an alternative concept for image guided femto second laser (fs-laser) surgery in the posterior eye with wavefront sensorless adaptive optics (WFSLAO). Our laboratory setup consists of an $800 \mathrm{~nm}$ fs-laser and a superluminescent diode (SLD) with $897.2 \mathrm{~nm}$ central wavelength. The SLD is used for optical coherence tomography (OCT) whereby the light for the OCT sample arm and the fs-laser share the same optical path which contains a deformable mirror, scanner and focusing optics. Energy calibrated photodiodes are used to measure the threshold energy for a laser induced optical breakdown inside a water filled chamber that acts as simple eye model. OCT image based metrics were used to determine a set of Zernike polynomials that describe a near optimal deformable mirror state. Such a mirror state improved OCT resolution and at the same time lowered the required fs-laser energy for a laser induced optical breakdown inside the eye model substantially.
\end{abstract}

Keywords: ophthalmic laser surgery, fs-laser, wavefront sensorless adaptive optics, optical coherence tomography

\section{INTRODUCTION}

Retinal detachment (RD) remains an important cause of visual loss. ${ }^{1}$ In the most common rhegmatogenous $\mathrm{RD}$, fluid is getting beneath the posterior retina, causing it to peel away from its normal position. In tractional $\mathrm{RD}$, typically caused by epiretinal membranes (EPMs) (macular pucker) due to diabetes, scarring detaches the retina. ${ }^{2}$ In both cases the RD leads to vitreous floaters. ${ }^{3}$ All these diseases can benefit from pars plana vitrectomy $(\mathrm{PPV})$, the conventional surgical treatment ${ }^{4}$ in which three instruments are brought into the posterior eye under visual control using a surgical microscope. Instead of using invasive instruments, which can result in cataract, ${ }^{5}$ less invasive laser systems can be applied to segment EPMs or treat symptomatic vitreous floaters ${ }^{6,7}$ without mechanically opening the eye. Until now, fs-lasers have only been used clinically in the anterior eye segment. The application of fs-lasers in the posterior eye segment still lacks the necessary accuracy, safety and effectiveness. ${ }^{8}$ Because of the relatively large aberrations in the human eye ${ }^{9}$ adaptive optics (AO) is indispensable to reduce the laser power for fs-laser treatments close to the light sensitive posterior retina. ${ }^{10}$ In traditional AO systems, originally invented for astronomical purposes, a wavefront sensor (e.g. Hartman-Shack-Sensor, HSS) is used to control an active optical element, like a deformable mirror (DM) in a closed loop to compensate the occurring phase aberrations in the analyzed wavefront. Traditional adaptive optics are designed for direct measurement of the wavefront in real time, which is achieved by a feedback loop controlling a deformable mirror. In wavefront

\# Both authors contributed equally to this work.

* Send correspondence to: m.zabic@lzh.de

Adaptive Optics and Wavefront Control for Biological Systems V, edited by Thomas G. Bifano, Sylvain Gigan, Na Ji, Proc. of SPIE Vol. 10886, 1088603 - @ 2019 SPIE · CCC code: 1605-7422/19/\$18 · doi: 10.1117/12.2509363 
sensorless adaptive optics, the deformable mirror is varied by certain algorithms and the optimization is carried out on the basis of a measured variable via a defined metric (e.g. image sharpness, intensity, etc.). ${ }^{11}$ The advantages of sensorless adaptive optics are the lower costs due to the elimination of the wavefront sensor and the reduced complexity of the setup, achieved by different aberration correction algorithms and metrics. ${ }^{12,13}$ There are a lot of successful applications for AO, in the field of microscopy ${ }^{14}$ or ophthalmology, where AO has been combined with OCT for retinal imaging. ${ }^{15}$ In the past years sensorless configurations have been published for high resolution imaging in mice ${ }^{16}$ and human ${ }^{17-20}$ eyes. The first sensorless AO algorithm approach was based on an image of an integrated camera sensor, ${ }^{21}$ which then was reduced to a simple photodetector. ${ }^{12}$ Since then, several approaches which utilizes OCT imaging have been published, mainly by using an image quality metric based on the intensity of en face images. ${ }^{16}$ But there have also been other approaches with different image metrics. ${ }^{22}$ In interferometric synthetic aperture microscopy (ISAM), the defocus for all depths can be corrected, but without considering the aberrations of the incident beam. ${ }^{23}$ Applying this computational adaptive optics principle on OCT images, it is possible to correct all aberrations for the imaging beam in postdata-acquisition, ${ }^{22,24}$ but not necessarily for a surgical laser beam. Furthermore, AO techniques with a novel multi-actuator adaptive lens instead of deformable mirror to control wavefront have been published recently. ${ }^{25}$ In this paper we present an approach to improve the focus of a surgical fs-laser for treatments in the deeper eye with wavefront sensorless adaptive optics (WFSLAO), based on the intensity of OCT images.

\section{MATERIALS AND METHODS}

\subsection{System Overview}

A schematic diagram of the laboratory setup for AO-assisted and OCT-guided fs-laser surgical instrument is depicted in figure 1. It is an advanced version of our previous setup ${ }^{8}$ that did not contain a liquid lens.

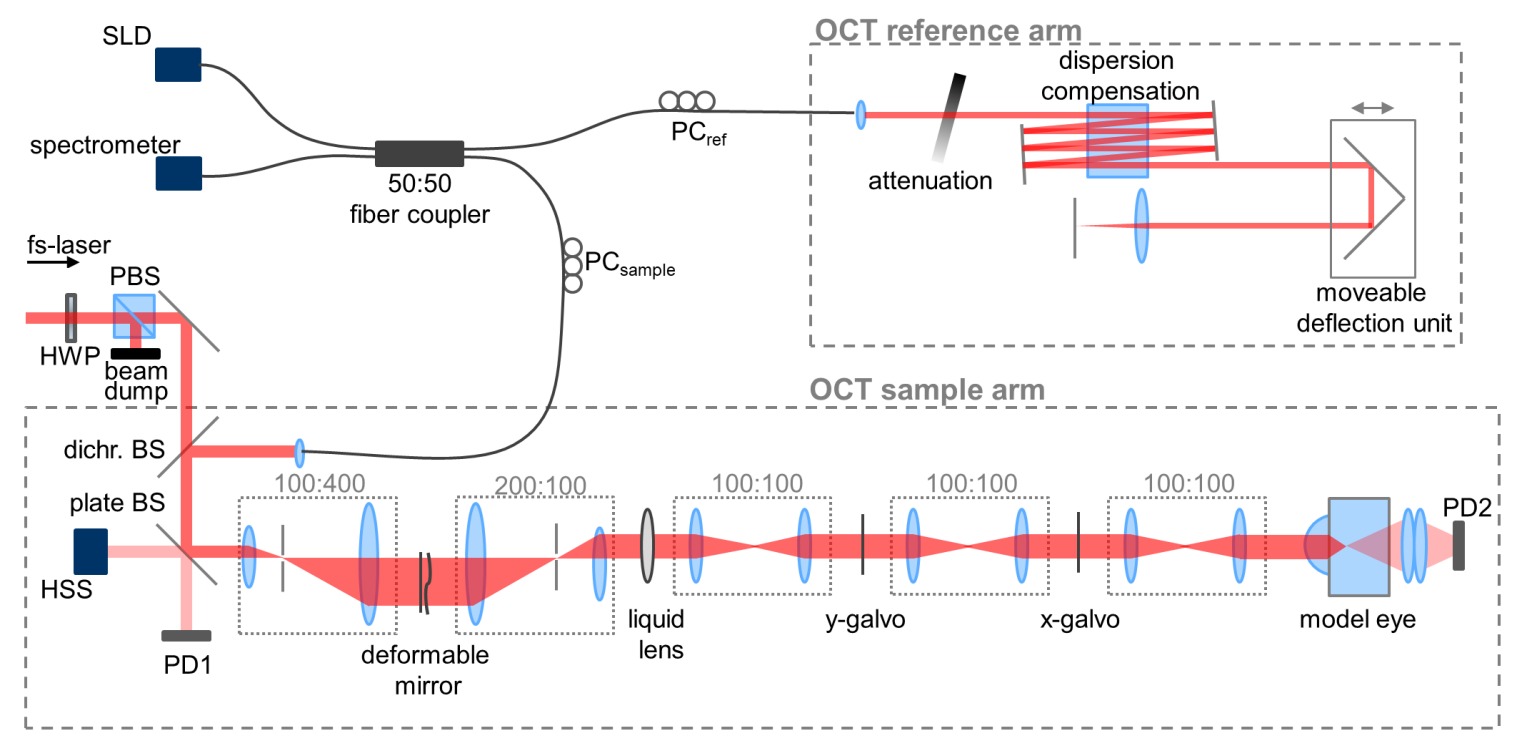

Figure 1: Unfolded beam path of the adaptive optics (AO) assisted fs-laser system extended by spectral domain optical coherence tomography (OCT) system for image-guided cutting in an eye model. SLD: superluminescent

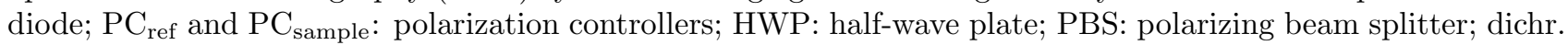
BS: dichroic beam splitter; plate BS: plate beam splitter; HSS: Hartmann-Shack wavefront sensor; PD1 and PD2: energy calibrated photodiodes.

The fiber coupled light source for OCT $\left(\lambda_{c}=897.2 \mathrm{~nm}, \Delta \lambda=151.7 \mathrm{~nm}\right.$, Broadlighter D-890-HP, Superlum, Ireland) internally combines two SLD modules which are protected from optical feedback by an optical isolator. The light enters a fiber based Michelson interferometer with splitting ratio of 50:50 and polarization controllers (PC) in both arms. The reference arm contains dispersion compensation glasses, folding mirrors, a variable 
neutral density filter, a movable deflection unit to adjust path length difference and a reflecting end mirror. The beam reflections from sample and reference arm are superimposed into a fiber coupled spectrometer (Custom Cobra Spectrometer with e2v EV71YEM4CL2010-BA8 camera, Wasatch Photonics, USA).

A Ti:sapphire fs-laser system $\left(\lambda_{c}=800 \mathrm{~nm}\right.$, Empower: pump, MaiTai: seed, Spitfire: amplifier, Spectra Physics, USA) with a pulse width of $147 \mathrm{fs}$ (FWHM of assumed gaussian pulse profile, at entrance of eye model) and a repetition rate of $5 \mathrm{kHz}$ is used as cutting laser. To control the incident laser energy, a motorized (PRM1/MZ rotational stage with TDC001 motor controller, Thorlabs, USA) half-wave plate (HWP) in combination with a polarizing beam splitter (PBS) is utilized. A dichroic beam splitter enables the coupling of fs-laser and SLD beam into the sample arm. The beam path for the SLD light and the fs-laser is nearly identical within the sample arm. A plate beam splitter followed by an energy calibrated photo diode (PD10 photo diode with Pulsar-2 pc interface, Ophir Optronics Solutions, Israel) placed in transmission direction allows to measure a known fraction of the incident fs-laser pulse energy. To adjust the beam diameter to the optical aperture of the deformable mirror (DM, mirao 52-e, Imagine Eyes, France), a 100:400 telescope is used. After the DM the beam is decreased in diameter and relayed on to a liquid lens (EL-10-30-Ci-NIR-LD-MV, Optotune, Switzerland) with a 200:100 telescope. Both telescopes consist of achromatic lenses to reduce aberrations, contain confocal pinholes and are passed in off-axis configuration to exclude unwanted back reflections. The liquid lens is positioned horizontally to avoid asymmetrical deformation of the lens due to gravity. The following 100:100 telescopes consist of customized lenses designed for diffraction limited quality up to $\pm 3.5^{\circ}$ optical scan angle. These telescopes relay the beam on $\mathrm{x}$-y-galvanometer scanners (GVS011, Thorlabs, USA) and finally on the entrance pupil of an eye model which consists of a 3D printed, distilled water filled chamber with an aspherical entrance lens.

To measure the optical wavefront or use closed loop adaptive optics a diffuse reflecting target (e.g. piece of PTFE tape) can be placed inside the eye model such that the SLD beam or the fs-laser is focused on it. The backscattered light is directed to the HSS (HASO3-first, Imagine Eyes, France) that enables wavefront measurement at the entrance pupil of the eye model. Through the telescopes the HSS is optically conjugated to eye model, x-galvanometer scanner, y-galvanometer scanner, liquid lens and deformable mirror.

A second energy calibrated photo diode (PD10 photo diode with Pulsar-2 pc interface, Ophir Optronics Solutions, Israel) is placed behind the eye model and measures the transmitted laser pulse energy. Measurements of both photo diodes are used to determine the threshold energy for a laser induced optical breakdown.

\subsection{Adaptive Optics without Wavefront Sensor}

Due to optical conjugation of the eye model pupil, x-y-scanners, liquid lens and deformable mirror with the wavefront sensor conventional adaptive optics wavefront measurement and closed loop control can be performed. In previous works ${ }^{26}$ we have already demonstrated that by measuring the wavefront and reshaping accordingly the DM to generate an nearly flat wavefront lateral OCT resolution increases and the necessary laser pulse energy for a laser induced optical breakdown in the eye model decreases. The improvement of lateral OCT resolution can also be achieved by wavefront sensorless approaches. ${ }^{16,18,20}$ The main challenges hereby are to find an appropriate image metric and a strategy for wavefront modulation. The following subsections give a brief overview of our developed software for WFSLAO, the image quality metric that we used as objective functions for optimization and finally we present the used optimization algorithm.

\subsubsection{Software overview}

The WFSLAO-software was implemented in our in-house laboratory framework "smartLab" which is written in $\mathrm{C}++$ and utilizes the cross-platform application framework Qt for the graphical user interface. Main feature of smartLab is the separation of hardware control (in so called "plug-ins") and application logic (in so called "jobs"). This makes it possible to exchange hardware components of the same type within a setup without the need to change anything in the source code. The processing software for OCT was also implemented in this manner and is discussed in more detail in 8 and 26. Figure 2 shows a UML class diagram of the WFSLAO-software module, showing the relationships between the following classes: 


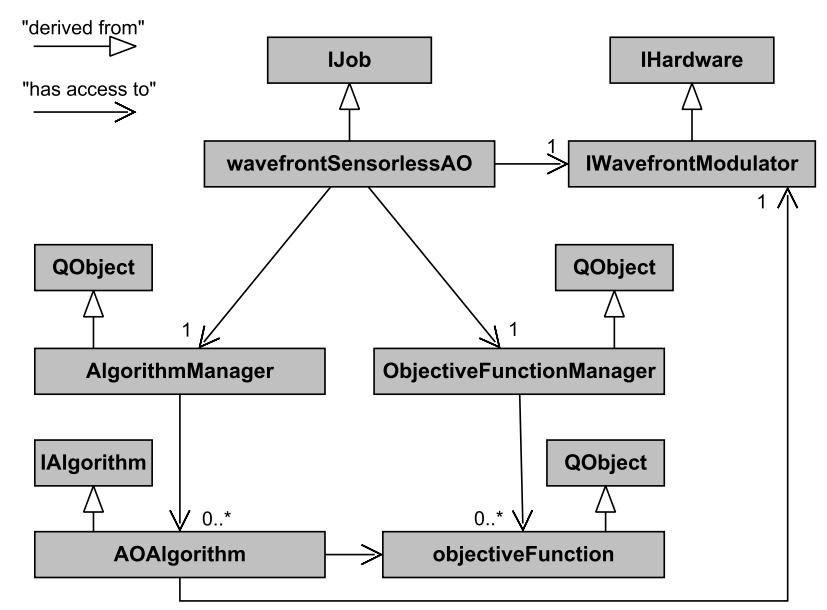

Figure 2: Class diagram of the WFSLAO software module with the most important classes and their relationship to each other.

IJob: smartLab interface for jobs. Jobs are small programs that can access the functionality of one or more smartLab plug-ins to perform a specific task. The interface specifies methods and attributes that every implementation of a job must have.

IHardware: smartLab interface for hardware plug-ins are individually loadable modules that can control hardware components. The interface specifies methods and attributes that every implementation of a smartLab plug-in must have.

wavefrontSensorlessAO: Job for the wavefront sensorless AO, derived from IJob. Knows which algorithms and objective functions are available and which algorithm can work with which objective function. Gives the active optimization algorithm access to the deformable mirror plug-in. Is responsible for the graphical user interface.

IWavefrontModulator: Base class of the plug-in for the deformable mirror. Enables communication between job and mirror plug-in.

QObject: Qt base class. Provides signal slot mechanism.

AlgorithmManager: Knows and owns all existing algorithms and can return a single or all algorithms on demand.

ObjectiveFunctionManager: Knows and owns all existing objective functions and can return a single or all objective functions on demand.

IAlgorithm: smartLab interface for algorithms. All IAlgorithm-derived classes can be managed by the AlgorithmManager.

AOAlgorithm: Base class for optimization algorithms of wavefront sensorless adaptive optics; derived from IAlgorithm. Each optimization algorithm (i.e. each AOAlgorithm) can be assigned an objective function (i.e. instance of objectiveFunction). Furthermore, the active optimization algorithm gets access to the deformable mirror at the start of the optimization process by wavefrontSensorlessAO.

objectiveFunction: Base class for objective functions. The objective function is the function that should be minimized or maximized. Instances of this class provide on demand a numerical value (e.g. sum of pixel values from specific area in a B-scan) that can be manipulated by changing the state of the deformable mirror.

The biggest advantage of this modular approach is the separation of optimization algorithm and objective function. It is possible to code both independently and link each optimization algorithm to any objective function. The actual procedure for determining or calculating the objective function value can be of any kind and can also 
access any program parts (auxiliary classes, jobs, plug-ins). This is crucial as it allows existing smartLab jobs and plug-ins to be used for the objective function.

\subsubsection{Objective functions for wavefront sensorless image optimization}

To determine applicable image metrics, i.e. objective functions for WFSLAO, we minimized existing wavefront errors and then purposely deteriorated the OCT image quality by stepwise applying different Zernike polynomials on the deformable mirror. For every mirror deformation B-scans of a resolution target (APL-OP01, Arden Photonics, UK) were acquired. The saved B-scans were used to calculate different image metrics for known wavefront aberrations. To minimize existing wavefront errors conventional AO with the software Casao 1.0 (Imagine Eyes, France) was used. The same software was used to perform open loop mirror deformation with Zernike polynomials. We separately applied seven Zernike polynomials with eleven $0.2 \mu \mathrm{m}$ steps from $-2.0 \mu \mathrm{m}$ to $2.0 \mu \mathrm{m}$ each. Here we want to present the results of the intensity metric. The intensity metric is calculated as sum of pixel values from a user defined image region. Figure 3 shows the metric values for different astigmatism (i.e. Zernike polynomial with Noll index 6) values; the corresponding B-scans, including red marks of the user definde image region, for RMS of $0.0 \mu \mathrm{m}$ and RMS of $2.0 \mu \mathrm{m}$ are depicted on the right side of the figure.
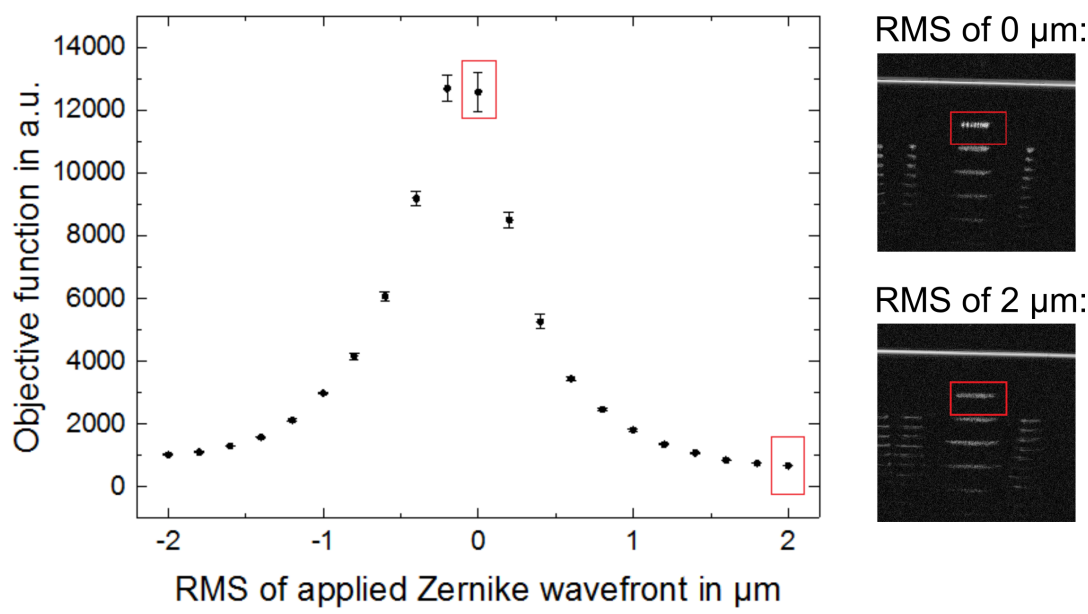

RMS of applied Zernike wavefront in $\mu \mathrm{m}$

Figure 3: Left: Objective function values for differend RMS values of applied Zernike polynomial shaped wavefront. Intensity metric was used as objective function and as Zernike polynomial astigmatism was used. Right: B-scans of OCT resolution target from Arden Photonics, with red marked region of interest, for $0.0 \mu \mathrm{m}$ RMS and $2.0 \mu \mathrm{m}$ RMS.

\subsubsection{Optimization Algorithm}

To find a mirror shape that maximizes an objective function, i.e. value of the intensity metric, we used a set of Zernike polynomials for mirror deformation. As an interaction matrix measurement was not performed the values of Zernike polynomials represented directly DM actuator voltages. The utilized DM has 52 actuators which are responsible for surface deformation. By setting an actuator voltage to a value from $-1 \mathrm{~V}$ to $1 \mathrm{~V}$ the mirror surface deforms inwards or outwards over a large area centered at the corresponding actuators position. This means that by applying any polynomial directly as actuator voltages to the mirror, the resulting mirror deformation does not exactly resemble the polynomial but still can be used for our purpose. A simple maximum find algorithm with reduction of search range and step size was used to search for optimal Zernike coefficients. Algorithm 1 shows the corresponding pseudo code of the utilized optimization algorithm. After every deformation of the mirror a wait time of $50 \mathrm{~ms}$ was introduced before evaluating object function value to take account of possible mirror oscillations. As input parameters we used zernikePolynomial = all polynomials from Noll index 0 to Noll index 56, minStepSize $=0.0001$, maxSamples $=11$, rangeReduction $=0.1$. 


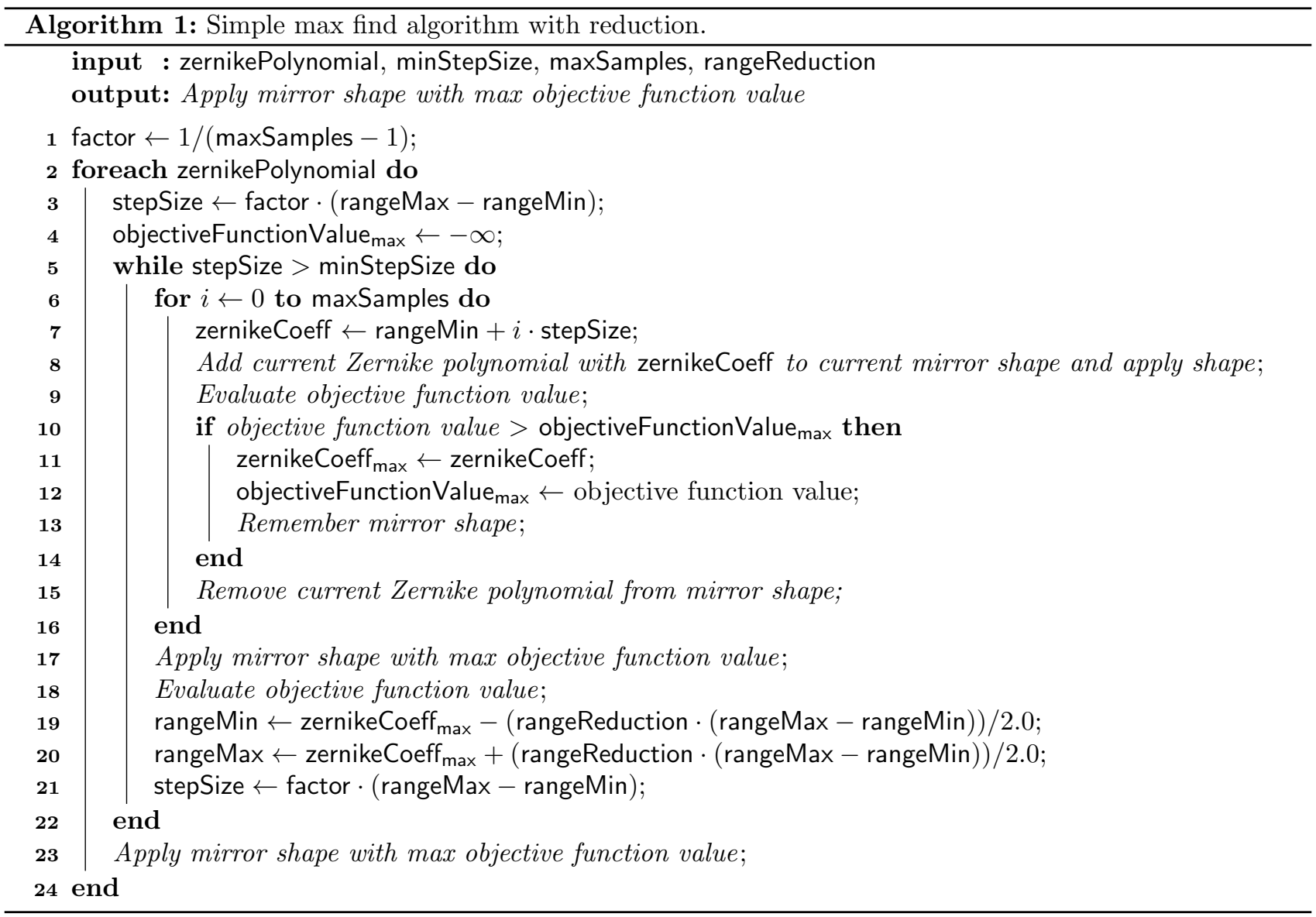

\section{RESULTS AND DISCUSSION}

To compare the resolution of the OCT system without AO, with coventional AO and with wavefront sensorless $\mathrm{AO}$ a resolution target (APL-OP01, Arden Photonics, UK) was scanned. OCT volume scans with 512 B-scans, each containing 512 A-scans, of the first group of the lateral resolution pattern were acquired. The stacked $\mathrm{B}$-scans were resliced with $\mathrm{Fiji}^{27}$ and a region of interest with 15 depth layers was averaged to generate the en face view. Figure 4 shows the cropped en face view of the lateral resolution pattern for a) the aberrated case, i.e. no $\mathrm{AO}$ is used and the $\mathrm{DM}$ is in flat state, $\mathrm{b}$ ) the conventional $\mathrm{AO}$ case, i.e. conventional measurement of the wavefront was performed and then corrected in open loop mode, c) the wavefront sensorless AO case, i.e. after intensity metric maximization according to Algorithm 1. The distance between the 11 lines in the resolution pattern decrease linearly from right to left from $10 \mu \mathrm{m}$ to $1 \mu \mathrm{m}$ in $1 \mu \mathrm{m}$ steps. The lateral resolution improved with conventional AO as well as with WFSLAO compared to the aberrated case as visible in figure 4 (d).

With WFSLAO the required fs-laser energy for a laser induced optical breakdown inside the distilled water filled eye model was reduced from $1.55 \mu \mathrm{J}$ (aberrated case, no AO utilized) to $0.48 \mu \mathrm{J}$. The LIOB threshold energy after conventional $\mathrm{AO}$ was determined to be $0.24 \mu \mathrm{J}$. Figure 5 shows the corresponding transmission measurements. 
(a) Aberrated

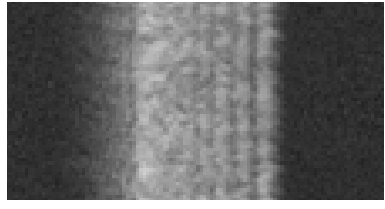

(b) Conventional $\mathrm{AO}$

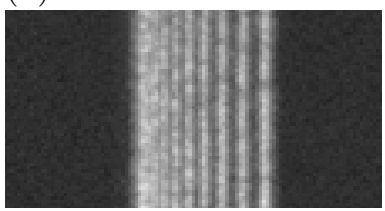

(c) WFSLAO

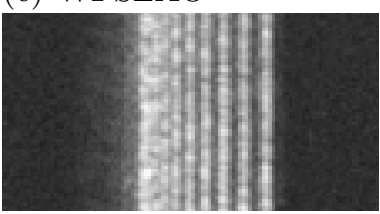

(d)

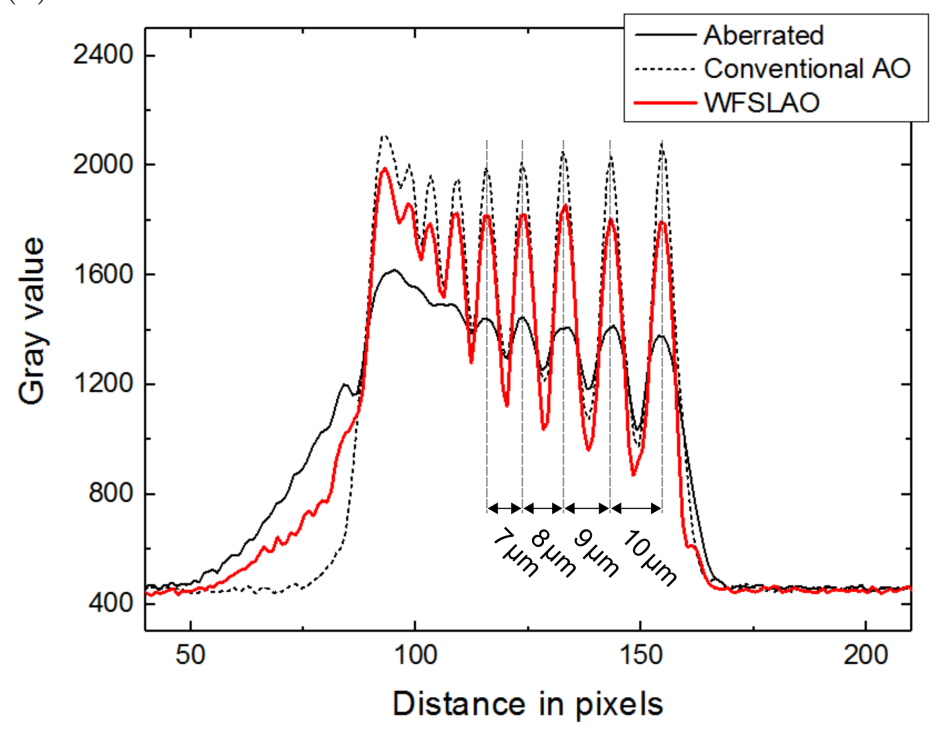

Figure 4: (a), (b), (c) En face views of lateral resolution pattern of OCT resolution target from Arden Photonics. The distance between the 11 lines decreases linearly from right to left from $10 \mu \mathrm{m}$ to $1 \mu \mathrm{m}$ in $1 \mu \mathrm{m}$ steps. (d) Profile plot of the averaged horizontal lines for each en face image.

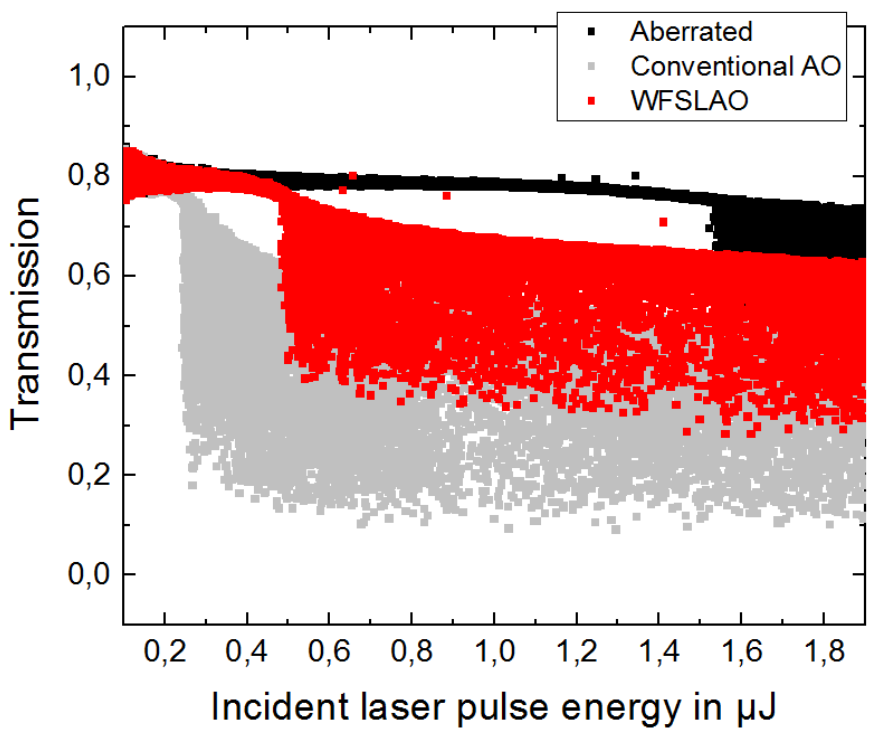

Figure 5: Transmission behavior of fs-laser pulses with different pulse energies for (a) aberrated, with (b) conventional AO and with (c) WFSLAO. Transmission decreases drastically as soon laser induced optical breakdown occurs.

\section{CONCLUSION}

We experimentally demonstrated that maximizing the overall intensity of a user defined region within acquired B-scans by optimizing the shape of a deformable mirror improves the lateral OCT resolution and at the same time decreases LIOB threshold energy substantially. Further investigations of cutting quality and optimization speed are necessary to determine if this approach is feasible for image-guided vitreo-retinal fs-laser surgery. Furthermore, the developed software framework enables us to use arbitrary objetive functions in combination 
with arbitrary optimization algorithms. This will allow us to use the LIOB threshold energy directly as objective function for future experiments.

\section{ACKNOWLEDGMENTS}

We acknowledge financial support for the project IKARUS (innovative cataract, age related presbyopia and retina treatment with ultrashort pulsed lasers; NO. 13N11847) by the German Federal Ministry of Education and Research (BMBF).

\section{REFERENCES}

[1] Schwartz, S. G. and Flynn, H. W., "Pars plana vitrectomy for primary rhegmatogenous retinal detachment," Clinical Ophthalmology 2(1), 57-63 (2008).

[2] Jalali, S., "Retinal detachment," Community Eye Health 16(46), 25-26 (2003).

[3] Stuart, A., "To treat - or not to treat - vitreous floaters," Eyenet Magazine, 31-33 (2018).

[4] Livingstone, B. I. and Bourke, R. D., "Retrospective study of macular holes treated with pars plana vitrectomy," Australian and New Zealand Journal of Ophthalmology 27, 331-341 (1999).

[5] Chung, C. P., Hsu, S. Y., and Wu, W. C., "Cataract formation after pars plana vitrectomy," The Kaohsiung journal of medical sciences 17, 84-89 (February 2001).

[6] Tsai, W. F., Chen, Y. C., and Su, C. Y., "Treatment of vitreous floaters with neodymium YAG laser," British Journal of Ophthalmology 77(8), 485-488 (1993).

[7] Shah, C. P. and Heier, J. S., "Yag laser vitreolysis vs sham yag vitreolysis for symptomatic vitreous floaters," JAMA Ophthalmology 135(9), 918-923 (2017). Medical study about YAG laser treatment of vitreous floaters.

[8] Matthias, B., Brockmann, D., Hansen, A., Horke, K., Knoop, G., Gewohn, T., Zabic, M., Krüger, A., and Ripken, T., "Concept for image-guided vitreo-retinal fs-laser surgery: adaptive optics and optical coherence tomography for laser beam shaping and positioning," in [Ophthalmic Technologies XXV], 9307, 93070Z, International Society for Optics and Photonics (2015).

[9] Salmon, T. O. and van de Pol, C., "Normal-eye zernike coefficients and root-mean-square wavefront errors," Journal of Cataract \& Refractive Surgery 32(12), 2064-2074 (2006).

[10] Hansen, A., Géneaux, R., Günther, A., Krüger, A., and Ripken, T., "Lowered threshold energy for femtosecond laser induced optical breakdown in a water based eye model by aberration correction with adpative optics," Biomed. Opt. Express 4(6), 852-867 (2013).

[11] Anzuola, E., Segel, M., Gladysz, S., and Stein, K., "Performance of wavefront-sensorless adaptive optics using modal and zonal correction," in [Optics in Atmospheric Propagation and Adaptive Systems XIX], Proceedings of SPIE 10002, 100020J (2016).

[12] Booth, M. J., "Wavefront sensorless adaptive optics for large aberrations," Optics Letters 32(1), 5-7 (2007).

[13] Lianghua, W., Yang, P., Kangjian, Y., Shanqiu, C., Shuai, W., Wenjing, L., and Xu, B., "Synchronous model-based approach for wavefront sensorless adaptive optics system," Optics Express 25(17), 20584-20597 (2017).

[14] Cua, M., Wahl, D. J., Zhao, Y., Lee, S., Bonora, S., Zawadzki, R. J., Jian, Y., and Sarunic, M. V., "Coherence-gated sensorless adaptive optics multiphoton retinal imaging," Scientific Reports 6(32223), 1-9 (2016).

[15] Zawadzki, R. J., Jones, S. M., Olivier, S. S., Zhao, M., Bower, B. A., Izatt, J. A., Choi, S., Laut, S., and Werner, J. S., "Adaptive-optics optical coherence tomography for high-resolution and high-speed 3D retinal in vivo imaging," Optics Express 13(21), 8532-8546 (2005).

[16] Jian, Y., Xu, J., Gradowski, M. A., Bonora, S., Zawadzki, R. J., and Sarunic, M. V., "Wavefront sensorless adaptive optics optical coherence tomography for in vivo retinal imaging in mice," Biomedical Optics Express 5(2), 547 (2014).

[17] Hofer, H., Sredar, N., Queener, H., Li, C., and Porter, J., "Wavefront sensorless adaptive optics ophthalmoscopy in the human eye," Optics Express 19(15), 14160-14171 (2011). 
[18] Wong, K. S. K., Jian, Y., Cua, M., Bonora, S., Zawadzki, R. J., and Sarunic, M. V., "In vivo imaging of human photoreceptor mosaic with wavefront sensorless adaptive optics optical coherence tomography," Biomedical Optics Express 6(2), 580-590 (2015).

[19] Jian, Y., Lee, S., Ju, M. J., Heisler, M., Ding, W., Zawadzki, R. J., Bonora, S., and Sarunic, M. V., "Lensbased wavefront sensorless adaptive optics swept source oct," Scientific Reports 6(27620), 1-10 (2016).

[20] Verstraete, H. R. G. W., Heisler, M., Ju, M. J., Wahl, D., Bliek, L., Kalkman, J., Bonora, S., Jian, Y., Verhaegen, M., and Sarunic, M. V., "Wavefront sensorless adaptive optics OCT with the DONE algorithm for in vivo human retinal imaging [invited]," Biomedical Optics Express 8(4), 2261-2275 (2017).

[21] Booth, M. J., Débarre, D., and Wilson, T., "Image-based wavefront sensorless adaptive optics," in [Advanced Wavefront Control: Methods, Devices, and Applications V], Proceedings of SPIE 6711, 671102 (2007).

[22] South, F. A., Liu, Y.-Z., Bower, A. J., Xu, Y., Carney, P. S., and Boppart, S. A., "Wavefront measurement using computational adaptive optics," Journal of the Optical Society of America A 35(3), 466-473 (2018).

[23] Adie, S. G., Graf, B. W., Ahmad, A., Darbarsyah, B., Boppart, S. A., and Carney, P. S., "The impact of aberrations on object reconstruction with interferometric synthetic aperture microscopy," in [Proceedings of SPIE], $\mathbf{7 8 8 9}$ (2011).

[24] Adie, S. G., Graf, B. W., Ahmad, A., Carney, P. S., and Boppart, S. A., "Computational adaptive optics for broadband optical interferometric tomography of biological tissue," PNAS 109(19), 7175-7180 (2012).

[25] Bonora, S., Jian, Y., Zhang, P., Zam, A., Pugh, E. N., Zawadzki, R. J., and Sarunic, M. V., "Wavefront correction and high-resolution in vivo oct imaging with an objective integrated multi-actuator adaptive lens," Optics Express 23(17), 21931-21941 (2015).

[26] Matthias, B., Zabic, M., Brockmann, D., Krüger, A., and Ripken, T., "Adaptive optics assisted and optical coherence tomography guided fs-laser system for ophthalmic surgery in the posterior eye," Journal of biomedical optics 21(12), 121512 (2016).

[27] Schindelin, J., Arganda-Carreras, I., Frise, E., Kaynig, V., Longair, M., Pietzsch, T., Preibisch, S., Rueden, C., Saalfeld, S., Schmid, B., et al., "Fiji: an open-source platform for biological-image analysis," Nature methods $\mathbf{9}(7), 676$ (2012). 